Europhys. Lett., 63 (2), pp. 296-302 (2003)

\title{
Nucleation of superconductivity in a mesoscopic rectangle
}

\author{
G. Teniers ${ }^{1}$, L. F. Chibotaru ${ }^{2}$, A. Cenlemans $^{2}$ and V. V. Moshchalkov $^{1}$ \\ 1 Katholieke Universiteit Leuven, Laboratorium voor Vaste-Stoffysica en Magnetisme \\ Celestijnenlaan 200D, 3001 Leuven, Belgium \\ 2 Katholieke Universiteit Leuven, Afdeling Kwantumchemie \\ Celestijnenlaan 200D, 3001 Leuven, Belgium
}

(received 26 February 2003; accepted in final form 20 May 2003)

PACS. 74.60.Ec - Mixed state, critical fields, and surface sheath.

PACS. 74.25. Dw - Superconductivity phase diagrams.

PACS. 74.20.De - Phenomenological theories (two-fluid, Ginzburg-Landau, etc.).

\begin{abstract}
We have studied the nucleation of superconductivity in a mesoscopic rectangle. We used an analytical gauge transformation for the vector potential $\boldsymbol{A}$ which gives $\boldsymbol{A}_{n}=0$ for the normal component along the boundary of the rectangle. Consequently, the linearized Ginzburg-Landau equation is reduced to an eigenvalue problem in the basis set of functions obeying the Neumann boundary condition. Through the application of this technique we are able to accurately determine the field-temperature superconducting phase boundary together with the corresponding vortex patterns. A range of aspect ratios for the rectangle has been investigated and compared with a superconducting square (aspect ratio $=1$ ) and with a superconducting line (aspect ratio $=\infty$ ). This also allows us to determine the stability of the vortex patterns with an anti-vortex in the centre, which have been predicted for a superconducting square, with respect to the deformation of the square.
\end{abstract}

Introduction. - Advances in microfabrication are creating new interesting opportunities for investigating the size and shape effects in mesoscopic superconducting structures $[1,2]$. The superconducting critical parameters can be improved by varying the shape and the size of a mesoscopic structure. However, not only the critical parameters are modified, also vortices do not necessarily arrange themselves in a traditional Abrikosov lattice [3-6]. For example, the superconducting mesoscopic square or triangle can have a symmetry-induced antivortex in the centre [7-10]. Interesting problems are posed by the rectangle geometry since a rectangle can be anything from a square to a line. For instance, the question is raised: how the vortex patterns in a square will alter when the aspect ratio $\zeta$ deviates from one and specifically what will happen with the symmetry-induced antivortex predicted for the square? On the other hand, it is interesting to investigate how the vortex patterns will evolve into the onedimensional pattern expected for the largest aspect ratios and how these vortex patterns are related to the field-temperature $(H-T)$ superconducting phase boundary.

The theoretical description of the superconductivity in an applied magnetic field requires the solution of the Ginzburg-Landau equations with the following boundary condition imposed 
on the order parameter $\Psi$ at the superconductor/vacuum interface [11]:

$$
\left.\left(-i \hbar \nabla-\frac{2 e}{c} \boldsymbol{A}\right) \Psi\right|_{n}=0
$$

where $\boldsymbol{A}$ is the vector potential corresponding to the magnetic field and $\Psi$ is the complex superconducting order parameter. To describe the nucleation of superconductivity at the $H-T$ phase boundary, it is sufficient to use the following linearized Ginzburg-Landau equation (LGL) [12], which is applicable for very small $|\Psi|$ :

$$
\frac{1}{2 m^{*}}\left(-i \hbar \nabla-\frac{2 e}{c} \boldsymbol{A}\right)^{2} \Psi=\frac{\hbar^{2}}{2 m^{*} \xi^{2}(T)} \Psi,
$$

where $\xi(T)$ is the temperature-dependent coherence length. Since $|\Psi|$ is very small at the phase boundary, the local field will be equal to the externally applied field. Furthermore the $z$-dependence (perpendicular to the rectangle cross-section and parallel to the magnetic field) disappears from the equations as long as demagnetisation effects and variations of $|\Psi|$ along the $z$-axis can be neglected. Therefore, when examining thin samples with a thickness smaller than the penetration depth $\lambda(T)$ and the coherence length $\xi(T)$, the problem is reduced to two dimensions. Also note that $\lambda(T)$ in itself does not appear in the LGL equation. Clearly, the use of the LGL equation already reduces the complexity of the problem since the LGL equation looks similar to the well-known Schrödinger equation for a free particle in an external magnetic field. However, the boundary condition (1) complicates matters severely. We use a special gauge transformation for the vector potential, which gives $\boldsymbol{A}_{n}=0$ for the normal component along the boundary line [13]. Then eq. (1) reduces to the Neumann boundary condition

$$
\left.\nabla \Psi\right|_{n}=0 \text {. }
$$

The gauge used for the rectangle geometry can be obtained from the gauge for the square geometry [13] by transforming the coordinates from $(x, y)$ to $(x \eta, y / \eta)$, where $\eta^{2}$ is the ratio of the length of the rectangle sides along the $x$ - and the $y$-axis, respectively.

The solutions of the LGL equation for the rectangle can then be characterized by the irreducible representations (irreps) A and B under the twofold rotation. This is due to the LGL equation being a linear equation, so any solution will have the same symmetry as the boundary. The lowest eigenvalue of the LGL equation gives the superconducting transition temperature as a function of magnetic field $T_{\mathrm{c}}(H)$. This lowest Landau level is, in fact, an alternation of two solutions belonging to the two irreps.

From square to rectangle. - When changing the aspect ratio of a rectangle from one, we should go from a fourfold to a twofold rotational symmetry. The lowest Landau level in a square follows a cusp-like profile corresponding to successive crossings of four different representations, it is reproduced in the rectangle with aspect ratio one by a pair of crossing irreps. However to obtain the solutions formed by the four lowest crossing solutions in a square, two pairs of crossing irreps are needed, not only the lowest solutions but also the first excited ones of the A and B symmetry. Departure from aspect ratio one leads to a splitting between the lowest and first-excited solutions. However, small deviations from the square have no noticeable effect on the lowest Landau level. All aspect ratios close to one have a lowest Landau level showing an oscillatory behaviour with a predominantly linear background dependence on the field.

At a field of 5 up to $6.3 \phi / \phi_{0}$ we find that the lowest Landau level corresponds to a solution with vorticity three. Although the energy levels and vorticity of all rectangles with an aspect 

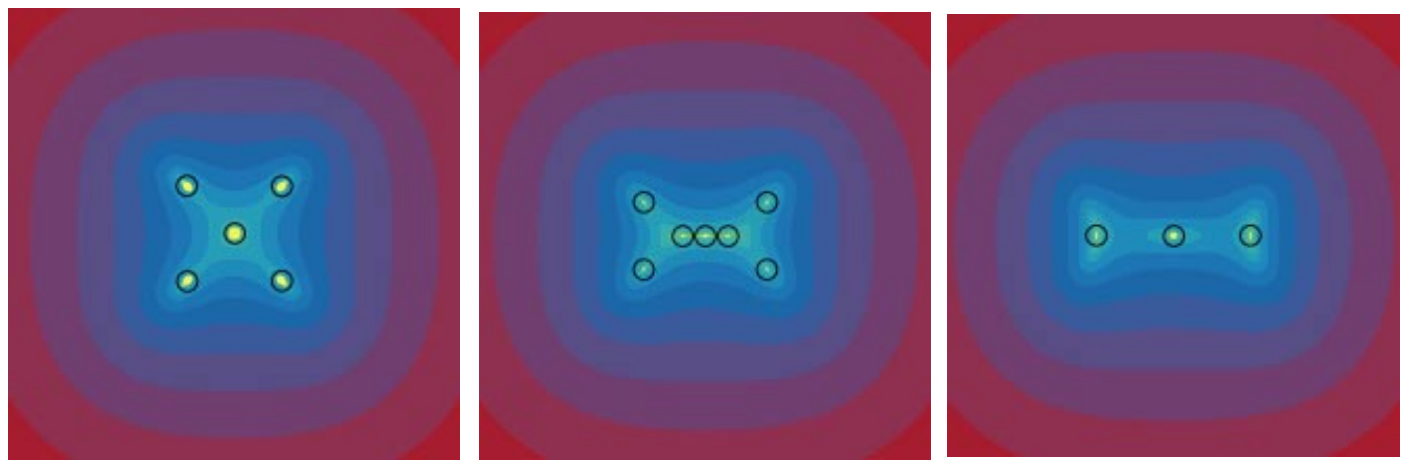

Fig. 1 - The figures show the density of the order parameter $|\psi|^{2}$ of the central $(a / 5) \times(b / 5)$ region in the rectangle, where $a$ is the long side and $b$ is the short side of the rectangle. The highest density is shown in red and the lowest $|\psi|^{2}$ values are coloured yellow, indicating the position of vortices and anti-vortices. Every color corresponds to roughly half an order of magnitude. At a field of $5.5 \phi / \phi_{0}$ the rectangles with, respectively, aspect ratio $1,1.01$ and 1.02, have very different vortex patterns. For aspect ratio one, we observe the known configuration with one antivortex in the centre and four vortices on the diagonals. However, at aspect ratio 1.01 we see four vortices on the diagonals, one in the centre and two anti-vortices next to the central vortex. And, finally, we find at aspect ratio 1.02 that there are three vortices positioned on the longest axis of the rectangle.

ratio very close to one are the same, the vortex pattern can nevertheless be very different (fig. 1). In a square, vorticity three is formed by four vortices on the diagonals and one antivortex in the centre. At an aspect ratio 1.02, vorticity three corresponds to three vortices on a row along the longest axis. However, when we consider an aspect ratio 1.01, which lies between these two values, it becomes even more complex. The vortex pattern is now built up from four vortices on the diagonals, one vortex in the centre and two anti-vortices located on the left and right of the central vortex along the long axis. This suggests the following evolution from aspect ratio 1 to aspect ratio 1.02. By varying the aspect ratio from one the anti-vortex in the centre splits into two anti-vortices plus one vortex in the centre. These anti-vortices move away from the centre along the long axis, where each anti-vortex merges with the pair of vortices respectively at the left and right of the anti-vortex, leaving, as a result, three vortices on a row parallel to the longest axis. We have to point out that the vortex pattern observed at aspect ratio 1.01 with a central vortex and two neighboring antivortices is in itself embedded in a region where the order parameter is already suppressed by orders of magnitude, which implies that we can also consider this structure as one elongated anti-vortex with a "strange" $(-1+1-$ 1) core structure. On top of that it is to be expected that this core structure is unstable when moving away from the $H-T$ phase boundary where the LGL equation is applicable, because the full GL equation does not require that the symmetry of the boundary should be preserved.

Rectangles with larger aspect ratios. - When investigating the solutions of eq. (2) (fig. 2), it is surprising to see that the $H$-T phase boundary remains almost the same for the aspect ratio between 1 and 1.333. However, the amplitude of the oscillations within the same irrep is reduced without substantial change in the lowest Landau level. Only when the aspect ratio becomes two or larger, a noticeable shift in the lowest Landau level is seen. Except for the first cusp the lowest Landau level still shows an oscillatory behaviour on top of a linear dependence on the field, though with a reduced slope. At an aspect ratio of four we can hardly observe the crossing of the different irreps as they almost completely merge together, only the first 

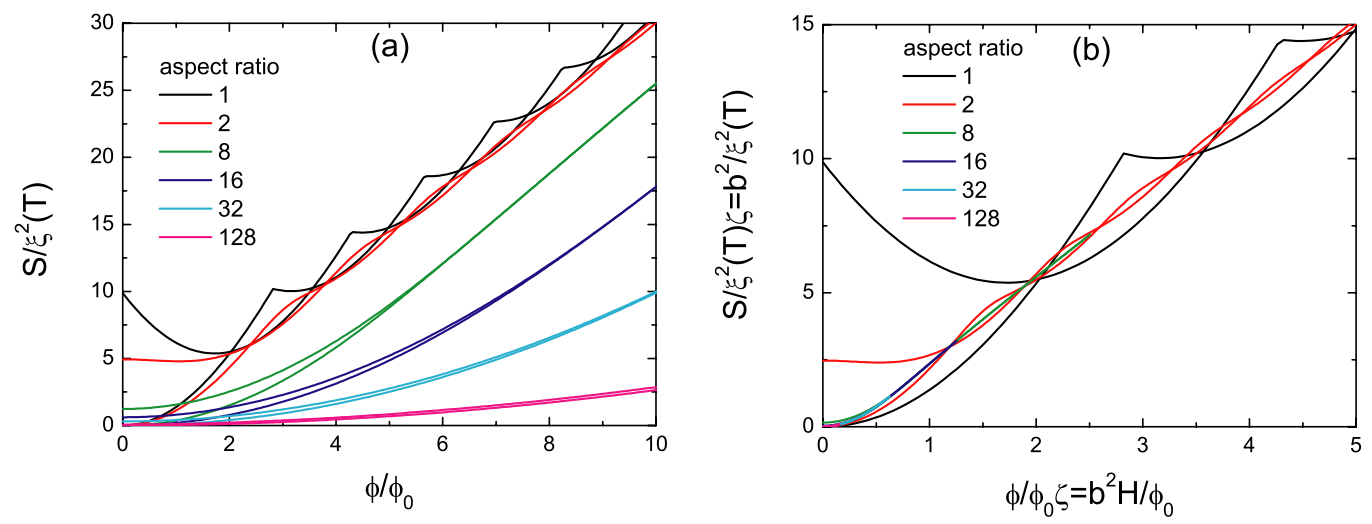

Fig. 2 - (a) Calculated superconducting $H-T$ phase boundary for rectangles with different aspect ratios. The superconducting phase is located above (lower $T$ ) and the normal phase below the $H$ - $T$ phase boundary. For every aspect ratio the lowest two eigenvalues of the LGL equation for the mesoscopic rectangles with superconductor-vacuum boundary conditions as a function of magnetic flux $\phi / \phi_{0}$ are shown, with the lowest eigenvalue corresponding to the phase boundary. The different colours correspond to different aspect ratios, as indicated in the figure, however with the same surface $S$. The lowest graph at zero field for the same colour is the solution for irrep A and the highest graph is the solution for irrep B. The flux is defined as $\phi=H S$ with $S$ the surface of the rectangle, $H$ the applied magnetic field and $\phi_{0}=h / 2 e c$ the superconducting flux quantum. (b) Different $H-T$ phase boundaries show fundamentally the same behaviour in a different field and temperature range, by scaling the different curves with the aspect ratio $\zeta$ onto the same universal curve, apart from the oscillations.

cusp remains clearly visible. For fields higher than the first crossing of solutions, the field dependence becomes again linear. The same pattern persists for even higher aspect ratios. However, the field at which the solutions for the first time cross increases with the aspect ratio (fig. 3). This crossing of the two irreps corresponds to the appearance of the first vortex in the rectangle, because irrep B describes solutions that have a vortex in the centre, while irrep A solutions are without a vortex in the centre. As a consequence, every crossing of the solutions

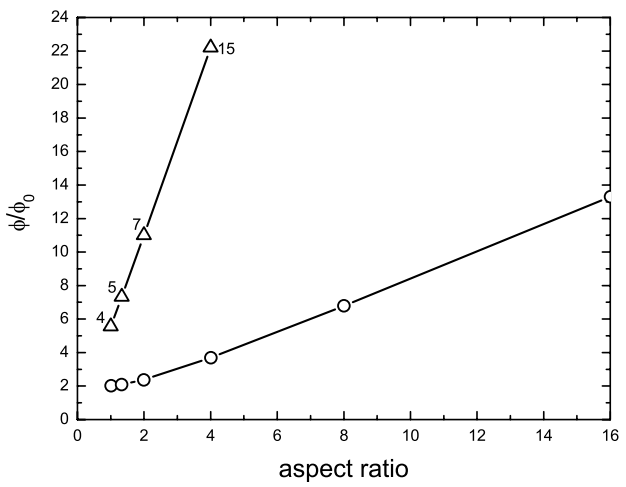

Fig. 3 - The circles show the flux at which the first vortex enters the rectangle. The open triangles are the first points on the $T_{\mathrm{c}}-H$ phase boundary, where vortices are located on the bisectors for the different aspect ratios. The numbers next to the squares indicate the corresponding number of vortices present in the rectangle at these fields. 

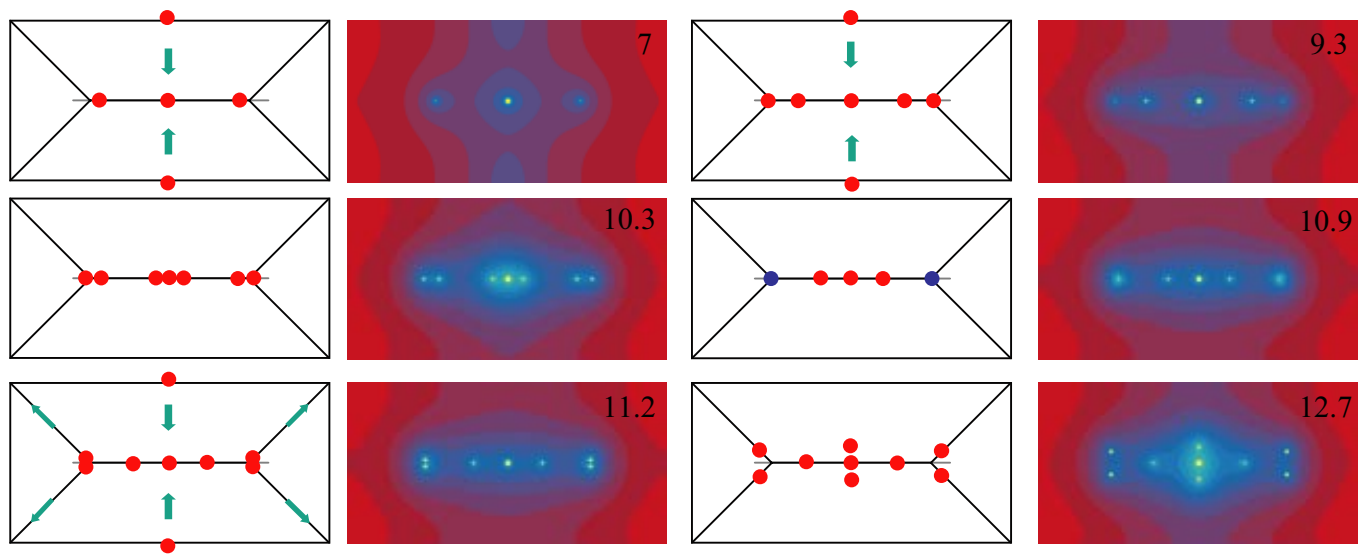

$+1 \phi_{0} \bullet+2 \phi_{0}$

Fig. 4 - The figures show the evolution of the vortex pattern in the rectangle with aspect ratio two while following a single solution corresponding to irrep B with increasing field. The same colour conventions as in fig. 1 were used. The numbers indicate the flux in the rectangle in units of the flux quantum $\phi_{0}$. The black lines are constructed by dividing the long axis with length $a$ in three regions. Both outer regions have a length of $b / 2$. If we removed the centre region, a square would remain with the black lines forming its diagonals.

corresponding to the two irreps will change the vorticity by one. The $H$ - $T$ phase boundaries for the different aspect ratios not only show the same behaviour, they additionally can be scaled on one universal curve, apart from the oscillations (fig. 2). The scaling is performed by dividing $S / \xi^{2}(T)$ and $\phi / \phi_{0}$ by the aspect ratio $\zeta$ :

$$
\frac{a b}{\xi^{2}(T) \zeta}=\frac{b^{2}}{\xi^{2}(T)}, \quad \frac{\phi}{\phi_{0} \zeta}=\frac{a b H}{\phi_{0} \zeta}=\frac{b^{2} H}{\phi_{0}} .
$$

Therefore, we can conclude that the length $b$ of the shortest side of the rectangle controls to a large part the behaviour of the superconducting mesoscopic rectangle, such as the entry of the first vortex and the slope of the phase boundaries.

In the case of aspect ratio 1.333 the vortices are situated in the central region of the longest axis of the rectangle. However, when the vorticity equals four, the vortices are sitting very close together. As a consequence, the vortices move away from the axis and instead are placed on the bisectors, which go from the corners to the long axis of the rectangle (see also fig. 4). The subsequent vortices appear again on the longest axis until the vorticity becomes twelve. At this point another four vortices move away from the axis towards the corners along the bisectors. This, of course, is in contrast to the square where all the vortices are always on the diagonals at the $H-T$ phase boundary.

The preference for positioning the vortices on the bisectors originates from the observed Meissner currents which make a hard bent there up to the longest axis (very weak screening), therefore the kinetic energy of the supercurrents is lower when a vortex is present on these bisectors compared to when a vortex is positioned away from the bisectors and the central region of the longest axis. A similar pattern of Meissner currents leads to a discontinuity along the bisectors in the problem of the entry of flux lines in a rectangle away from the $H-T$ phase boundary observed by magneto-optical techniques [14-16]. 
The same scheme repeats itself for aspect ratio two; however, we have to wait until there are seven vortices in the rectangle before four vortices start to be located on the bisectors. For the aspect ratio four it takes 15 vortices to initiate the vortex redistribution from the axis to the bisectors. So it is clear that with growing aspect ratio the field at which the vortices start to take positions on the bisectors, instead of the longest axis, increases. From fig. 3 we can see that the lowest field at which vortices appear first at the bisectors increases linearly with the aspect ratio for at least the lowest aspect ratios. For aspect ratios beyond four we cannot go high enough in field with a basis set of 1764 basis functions to see vortices on the bisectors. For the largest aspect ratios even the entrance of the first vortex in the rectangle is beyond the field range accessible by a limited number of basis functions in the set.

In summary, it has been shown that the vortex pattern in a rectangle is composed of a central row of vortices along the longest axis and the vortices that are situated on the bisectors. These bisectors span the left and right $b / 2$ region, where $b$ is the short side of the rectangle. The vortices forming a row parallel to the longest axis only move into these two outer regions when they are ready to reposition themselves onto the bisectors, as shown in fig. 4. When following the same irrep with increasing field (fig. 4), we can observe the vortices enter in pairs through the centers of the long edges. When more vortices accumulate on the central line, migration starts to the outer $b / 2$ regions. In this region on the longest axis, two pairs of vortices will merge at the two points where the bisectors cross and form two giant vortices with vorticity two. These giant vortices split again into separate vortices which move along the bisectors now.

Lines. - When increasing the aspect ratio to large values, the rectangle eventually resembles a line. The $H$-T phase boundary for a line with a width smaller than the coherence length $\xi(T)$ in a perpendicular magnetic field is well known for the London limit, since it coincides with the problem of a thin $(\ll \xi(T))$ superconducting plane in a parallel magnetic field $[2,17]$ :

$$
\frac{1}{\xi^{2}(T)}=\frac{\pi^{2} w^{2} H^{2}}{3 \phi_{0}^{2}}
$$

We can get an approximation for the rectangle with large aspect ratio when we substitute the width $w^{2}$ with $b^{2}=a b / \zeta$, where $\zeta$ is the aspect ratio, $a$ is the long side and $b$ is the short side of the rectangle,

$$
\frac{a b}{\xi^{2}(T)}=\frac{\pi^{2}}{3 \zeta}\left(\frac{\phi}{\phi_{0}}\right)^{2} .
$$

This solution, which is obtained by minimizing the GL free energy in the London limit, is only valid when $|\Psi|$ is approximately spatially constant. Consequently, the valid field region will increase with aspect ratio, since the field of the first entrance of a vortex increases with increasing aspect ratio. For instance, we determined that within the shown field region this approximation already coincides perfectly with our calculations (fig. 2) for a rectangle with aspect ratio 64 .

Conclusions. - In a mesoscopic rectangle the superconductivity nucleates by forming specific vortex patterns which consist of a one-dimensional element, namely a row along the longest axis. We also have found a two-dimensional vortex pattern, a bit similar to the vortex pattern in a square, where vortices are positioned on the diagonals. By changing the aspect ratio the vortex patterns are modified. To realise similar vortex patterns, the field has to be increased considerably with increasing aspect ratio. For example, the field at which the first vortex enters and the field at which the vortices move to the corners will rise significantly. For large aspect ratios the vortex pattern evolves from the typical two-dimensional pattern for a square to the one-dimensional pattern expected for a line. 
In addition, we have observed how the $H-T$ phase boundary changes when the aspect ratio increases and the square transforms into a line. First, the amplitude of the oscillations is reduced, then the slope is changed and finally the phase boundary evolves into a parabolic dependence on the field (the first cusp) which corresponds with the solution of a line.

Finally, we have found that the vortex pattern in a square with an antivortex in the centre is very sensitive to the deformation of the square. A deviation of two percent between the two axes of the square is sufficient to induce a deformation of the vortex pattern. This implies that any observation of this complex vortex molecule will also require a very precise sample preparation aside from a very sensitive and high-resolution measuring technique.

$$
* * *
$$

This work is supported by the Flemish Science Fund (FWO) and the Concerted Action Programme (GOA), the IUAP network of the Belgian Government, and by the ESF VORTEX Programme.

\section{REFERENCES}

[1] Bruyndoncx V., Rodrigo J. G., Puig T., van Look L. and Moshchalkov V. V., Phys. Rev. B, 60 (1999) 4285.

[2] Moshchalkov V., Gielen L., Strunk C., Jonckheere R., Qiu X., van Haesendonck C. and Bruynseraede Y., Nature, 373 (1995) 319.

[3] Moshchalkov V. V. et al., Handbook of Nanostructured Materials and Nanotechnology, edited by Nalwa H. S., Vol. 3 (Academic, San Diego) 1999, p. 9.

[4] Saint-James D., Phys. Lett., 15 (1965) 13.

[5] Fink H. J. and Presson A. G., Phys. Rev., 151 (1966) 219.

[6] Baelus B. J., Yampolskit S. V. and Peeters F. M., Phys. Rev. B, 66 (2002) 869.

[7] Chibotaru L. F., Ceulemans A., Bruyndoncx V. and Moshchalkov V. V., Nature, 408 (2000) 833.

[8] Chibotaru L. F. et al., Phys. Rev. Lett., 86 (2001) 1323.

[9] Bonca J. and Kabanov V. V., Phys. Rev. B, 65 (2002) 2509.

[10] Misko V. R., Fomin V. M., Devreese J. T. and Moshchalkov V. V., cond-mat/0203140.

[11] Saint-James D. and De Gennes P.-G., Phys. Lett., 7 (1963) 306.

[12] De Gennes P.-G., Superconductivity of Metals and Alloys (Benjamin, New York) 1966.

[13] Chibotaru L. F., Ceulemans A., Teniers G., Bruyndoncx V. and Moshchalkov V. V., Eur. Phys. J. B, 27 (2002) 341.

[14] Schuster Th., Indenbom M. V., Koblischka M. R., Kuhn H. and Kronmüller H., Phys. Rev. B, 49 (1994) 3443.

[15] Brandt E. H., Phys. Rev. B, 52 (1995) 15442.

[16] Schuster Th., Kuhn H. and Brandt E. H., Phys. Rev. B, 54 (1996) 3514.

[17] Tinkham M., Introduction to Superconductivity (McGraw Hill, New York) 1975. 